\title{
Existence of Solutions for a Fractional Laplacian Equation with Critical Nonlinearity
}

\author{
Zifei Shen and Fashun Gao \\ Department of Mathematics, Zhejiang Normal University, Jinhua 321004, China \\ Correspondence should be addressed to Zifei Shen; szf@zjnu.cn
}

Received 5 July 2013; Revised 11 October 2013; Accepted 18 October 2013

Academic Editor: Mihai Mihǎilescu

Copyright (c) 2013 Z. Shen and F. Gao. This is an open access article distributed under the Creative Commons Attribution License, which permits unrestricted use, distribution, and reproduction in any medium, provided the original work is properly cited.

We study the fractional Laplacian equation $(-\Delta)^{s} u+\lambda A(x) u=\mu u+|u|^{2^{*}(s)-2} u, x \in \mathbb{R}^{N}$, here $N>2 s, s \in(0,1), 2^{*}(s)=2 N /(N-2 s)$ is the critical exponent, and $A(x) \geq 0$ is a real potential function. Employing the variational method we prove the existence of nontrivial solutions for $\mu$ small and $\lambda$ large.

\section{Introduction}

We consider the nonlinear Schrödinger equation:

$$
i \hbar \frac{\partial \psi}{\partial t}=-\hbar^{2} \Delta \psi+A(x) \psi-|\psi|^{p-2} \psi, \quad x \in \mathbb{R}^{N}
$$

where $\hbar$ is the Planck constant. When looking for stationary waves of the form $\psi(t, x)=e^{-i \mu(\hbar t)} \varphi(x)$ with $\mu \in \mathbb{R}^{N}$, one is led to considering the elliptic equation in $\mathbb{R}^{N}$; namely, replacing $\hbar$ by $\varepsilon$, one sees that $\varphi$ must satisfy

$$
-\varepsilon^{2} \Delta \varphi+A(x) \varphi=\varepsilon^{2} \mu \varphi+|\varphi|^{p-2} \varphi .
$$

Setting $u(x):=\varepsilon^{-2 /(p-2)} \varphi(x)$ and $\lambda=\varepsilon^{-2}$, this equation is transformed into

$$
-\Delta u+\lambda A(x) u=\mu u+|u|^{2^{*}-2} u, \quad x \in \mathbb{R}^{N} .
$$

Problem (3) has been widely studied in the literature (see, for instance, $[1,2]$ and references therein), where $2^{*}=2 \mathrm{~N} /(\mathrm{N}-$ 2 ) is the critical exponent $N \geq 4$, and $A(x) \geq 0$ is a potential well.

The study of existence and concentration of the semiclassical states of Schrödinger equation goes back to the pioneer work [3] by Floer and Weinstein. Ever since then, equations of (3) type with subcritical nonlinearities $\left(p<2^{*}=2 \mathrm{~N} /(\mathrm{N}-2)\right.$ for $N \geq 3$ ) have been studied by many authors. For critical nonlinearity ( $p=2^{*}$ for $N \geq 4$ ), Clapp and Ding $[1,2]$ established the existence and multiplicity of positive solutions and minimal nodal solutions which localize near the potential well for $\mu$ small and $\lambda$ large.

The fractional Schrödinger equation is a fundamental equation of fractional quantum mechanics. It was discovered by Nick Laskin as a result of extending the Feynman path integral, from the Brownian-like to Lévy-like quantum mechanical paths. The term fractional Schrödinger equation was coined by Nick Laskin.

Recently, a great attention has been devoted to the fractional and nonlocal operators of elliptic type, both for their interesting theoretical structure and in view of concrete applications in many fields such as combustion and dislocations in mechanical systems. This type of operator seems to have a prevalent role in physical situations and has been studied by many authors [4-9] and references therein. In [5], Di Nezza et al. deal with the fractional Sobolev space $W^{s, p}$ and analyze their role in the trace theory. They prove continuous and compact embeddings, investigating the problem of the extension domains and other regularity results. In [8], Felmer et al. proved the existence of positive solutions of nonlinear Schrödinger equation involving the fractional Laplacian in $\mathbb{R}^{N}$. They further analyzed regularity, decay, and symmetry properties of these solutions. Servadei and Valdinoci [9] studied the existence of nontrivial solutions for equations driven by a nonlocal integrodifferential operator $L_{K}$ with homogeneous Dirichlet boundary conditions. 
They give more general and more precise results about the eigenvalues of a linear operator.

The aim of this paper is to study the fractional Laplacian equation:

$$
(-\Delta)^{s} u+\lambda A(x) u=\mu u+|u|^{2^{*}(s)-2} u \text { in } \mathbb{R}^{N},
$$

where $N>2 s, \lambda>0, \mu \in \mathbb{R}, s \in(0,1)$, and $H^{s}\left(\mathbb{R}^{N}\right)$ is the usual fractional Sobolev space, and $2^{*}(s)=2 N /(N-2 s)$ is the corresponding critical exponent. Suppose $A(x)$ satisfies the following assumptions.

(A1) $A \in C\left(\mathbb{R}^{N}, \mathbb{R}\right), A \geq 0, \Omega:=\operatorname{int} A^{-1}(0)$ is a nonempty bounded set with smooth boundary, and $\bar{\Omega}=A^{-1}(0)$.

(A2) There exists $M_{0}>0$ such that

$$
L\left\{x \in \mathbb{R}^{N}: A(x) \leq M_{0}\right\}<\infty,
$$

where $L$ denotes the Lebesgue measure in $\mathbb{R}^{N}$.

The fractional Laplace operator $(-\Delta)^{s}$ in (4) can be defined as

$$
\begin{aligned}
-(-\Delta)^{s} u(x) & \\
& =\frac{1}{2} \int_{\mathbb{R}^{N}} \frac{u(x+y)+u(x-y)-2 u(x)}{|y|^{N+2 s}} d y, \quad x \in \mathbb{R}^{N} .
\end{aligned}
$$

We say that a function $u \in H^{s}\left(\mathbb{R}^{N}\right)$ solves (4) in the weak sense if

$$
\begin{gathered}
\int_{\mathbb{R}^{2 N}} \frac{(u(x)-u(y))(\varphi(x)-\varphi(y))}{|x-y|^{N+2 s}} d x d y \\
+\lambda \int_{\mathbb{R}^{N}} A(x) u(x) \varphi(x) d x \\
=\mu \int_{\mathbb{R}^{N}} u(x) \varphi(x) d x \\
+\int_{\mathbb{R}^{N}}|u(x)|^{2^{*}(s)-2} u(x) \varphi(x) d x, \\
\forall \varphi \in H^{s}\left(\mathbb{R}^{N}\right) .
\end{gathered}
$$

Define the energy functional by

$$
\begin{aligned}
I_{\lambda}(u)= & \frac{1}{2} \int_{\mathbb{R}^{2 N}} \frac{|u(x)-u(y)|^{2}}{|x-y|^{N+2 s}} d x d y \\
& +\frac{1}{2} \lambda \int_{\mathbb{R}^{N}} A(x)|u(x)|^{2} d x \\
& -\frac{1}{2} \mu \int_{\mathbb{R}^{N}}|u(x)|^{2} d x-\frac{1}{2^{*}(s)} \int_{\mathbb{R}^{N}}|u(x)|^{2^{*}(s)} d x .
\end{aligned}
$$

Then we know the critical points of $I_{\lambda}$ are exactly the weak solutions of (7). In this sense we will prove the existence of the critical points of the functional $I_{\lambda}$. Fréchet derivative of $I_{\lambda}$ is

$$
\begin{aligned}
\left\langle I_{\lambda}^{\prime}(u), \varphi\right\rangle= & \int_{\mathbb{R}^{2 N}} \frac{(u(x)-u(y))(\varphi(x)-\varphi(y))}{|x-y|^{N+2 s}} d x d y \\
& +\lambda \int_{\mathbb{R}^{N}} A(x) u(x) \varphi(x) d x \\
& -\mu \int_{\mathbb{R}^{N}} u(x) \varphi(x) d x \\
& -\int_{\mathbb{R}^{N}}|u(x)|^{2^{*}(s)-2} u(x) \varphi(x) d x
\end{aligned}
$$$$
\forall \varphi \in H^{s}\left(\mathbb{R}^{N}\right)
$$

Concerning the Schrödinger equation:

$$
\begin{aligned}
-\Delta u+\lambda A(x) & =\mu u+|u|^{2^{*}-2} u \quad \text { in } \mathbb{R}^{N}, \\
u & >0 \quad \text { in } \mathbb{R}^{N}, \\
u & \in H^{1}\left(\mathbb{R}^{N}\right) .
\end{aligned}
$$

Clapp and Ding [1] proved the following.

(a) Assume $(A 1)$ and $(A 2)$ hold and $N \geq 4$. Then, for every $0<\mu<\mu_{1}^{1}(\Omega)$, there exists $\lambda(\mu)>0$ such that (4) has a least solution $u_{\lambda}$ for each $\lambda \geq \lambda(\mu)$, where $\mu_{1}^{1}(\Omega)$ is the first eigenvalue of $-\Delta$ on $\Omega$ with boundary condition $u=0$.

(b) Assume $(A 1)$ and $(A 2)$ hold and $N \geq 4$. Then, there exist $0<\mu^{*}<\mu_{1}^{1}(\Omega)$ and for each $0<\mu \leq \mu^{*}$ there exist two numbers $\Lambda(\mu)>0$ and $0<c(\mu)<$ $(1 / N) S^{N / 2}$ such that if $\lambda \geq \Lambda(\mu)$, then (4) has at least $\operatorname{cat}(\Omega)$ (the number of solutions is bounded from below by a topological invariant) solutions with energy $I_{\lambda, \mu} \leq c(\mu)$.

(c) Every sequence of solutions $\left(u_{n}\right)$ of (10) such that $0<$ $\mu<\mu_{1}^{1}(\Omega), \lambda_{n} \rightarrow \infty$ and $I_{\lambda_{n}, \mu}\left(u_{n}\right) \rightarrow c<(1 / N) S^{N / 2}$ as $n \rightarrow \infty$ concentrates at a solution of

$$
\begin{gathered}
-\Delta u=\mu u+|u|^{2^{*}-2} u \quad \text { in } \Omega, \\
u>0 \quad \text { in } \Omega, \\
u=0 \quad \text { on } \partial \Omega,
\end{gathered}
$$

where $S$ is the best Sobolev constant.

Our aim is to show that (a) and (c) can be extended to problem (4). In this paper, we have the following results.

Theorem 1. Assume (A1) and (A2) hold $N>2 s$ and $s \in(0,1)$. Then, for every $0<\mu<\mu_{1}(\Omega)$, there exists $\lambda(\mu)>0$ such that (4) has at least a solution $u$ for each $\lambda \geq \lambda(\mu)$, where $\mu_{1}(\Omega)$ is the first eigenvalue of $(-\Delta)^{s}$ on $\Omega$ with boundary condition 
$u=0$. There is a great deal of work on $\mu_{1}(\Omega)$; see for example [9]. We have

$\mu_{1}(\Omega)$

$$
=\inf _{u \in H_{0}^{s}(\Omega) \backslash\{0\}} \frac{\int_{\mathbb{R}^{2 N}}\left(|u(x)-u(y)|^{2} /|x-y|^{N+2 s}\right) d x d y}{\int_{\Omega}|u(x)|^{2} d x} .
$$

Theorem 2. Every sequence of solutions $\left(u_{n}\right)$ of (4) such that $0<\mu<\mu_{1}(\Omega), \lambda_{n} \rightarrow \infty$, and $I_{\lambda}\left(u_{n}\right) \rightarrow c<(s / N) S_{s}^{N / 2 s}$ as $n \rightarrow \infty$ concentrates at a solution of

$$
\begin{gathered}
(-\Delta)^{s} u=\mu u+|u|^{2^{*}(s)-2} u \quad \text { in } \Omega, \\
u=0 \text { on } \mathbb{R}^{N} \backslash \Omega,
\end{gathered}
$$

where $\Omega$ is defined as in $(A 1)$.

Here $S_{s}$ is defined as

$$
S_{s}:=\inf _{u \in E \backslash\{0\}} \frac{\int_{\mathbb{R}^{2 N}}\left(|u(x)-u(y)|^{2} /|x-y|^{N+2 s}\right) d x d y}{|u|_{2^{*}(s)}^{2}},
$$

where $E$ is an $L^{2}\left(\mathbb{R}^{N}\right)$ space with potential and will be defined in Section 2.

There is a great deal of work on (13); see, for example, [4, $6,7]$ and the references therein. Among them Servadei and Valdinoci $[4,6,7]$ studied the problem

$$
\begin{gathered}
L_{K} u+\lambda u+|u|^{2^{*}(s)-2} u+f(x, u)=0 \quad \text { in } \Omega, \\
u=0 \quad \text { in } \mathbb{R}^{N} \backslash \Omega,
\end{gathered}
$$

where $\Omega$ is an open bounded set with Lipschitz boundary in $\mathbb{R}^{N}, N>2 s, s \in(0,1), \lambda>0$ is a real parameter. $L_{K}$ is defined as follows:

$$
L_{K} u(x)=\frac{1}{2} \int_{\mathbb{R}^{N}}(u(x+y)+u(x-y)-u(x)) K(y) d x d y,
$$

$$
x \in \mathbb{R}^{N} \text {. }
$$

Here $K: \mathbb{R}^{N} \backslash\{0\} \rightarrow(0,+\infty)$ is a function such that

$$
m(x) \cdot K \in L^{1}\left(\mathbb{R}^{N}\right), \quad \text { where } m(x)=\min \left\{|x|^{2}, 1\right\} ;
$$

there exists $\theta>0$ such that $K(x) \geq \theta|x|^{-(N+2 s)}$ and $K(x)=$ $K(-x)$ for any $x \in \mathbb{R}^{N} \backslash\{0\}$. They proved that problem (15) admits a nontrivial solution for any $\lambda>0$. They also studied the case $f(x, u) \equiv 0$ and $K(x)=|x|^{-(N+2 s)}$, respectively.

Clapp and Ding [1] proved the existence of minimizing sequence for energy function of (10) on Nehari manifold and assumed that it is a Palais Smale sequence by Ekeland's variational principle. Since Palais Smale conditions hold, this finished the proof of (a). For (c), they analyzed the problem directly. We will show that their method can be extended to the case $0<s<1$.

This paper is organized as follows. In Section 2, we give some preliminary results. In Section 3, we finish the proof of Theorem 1. In Section 4, we finish the proof of Theorem 2.

\section{Preliminary Results}

Throughout this paper we write $|\cdot|_{q}$ for the $L^{q}$ norm for $q \epsilon$ $[1, \infty]$. We always assume that $(A 1)-(A 2)$ hold, $N>2 s, \lambda>0$, $\mu \in \mathbb{R}$, and $s \in(0,1) . \mu_{1}(\Omega)$ is the first eigenvalue of $(-\Delta)^{s}$ on $\Omega . \Omega$ is a nonempty bounded set with smooth boundary.

We consider the fractional Sobolev space:

$$
\begin{aligned}
& H^{s}\left(\mathbb{R}^{N}\right) \\
& =\left\{u \in L^{2}\left(\mathbb{R}^{N}\right) \mid \int_{\mathbb{R}^{2 N}} \frac{|u(x)-u(y)|^{2}}{|x-y|^{N+2 s}} d x d y\right. \\
& \left.\quad+\int_{\mathbb{R}^{N}}|u(x)|^{2} d x<+\infty\right\}
\end{aligned}
$$

with norm

$$
\|u\|_{H^{s}}=\left(\int_{\mathbb{R}^{2 N}} \frac{|u(x)-u(y)|^{2}}{|x-y|^{N+2 s}} d x d y+\int_{\mathbb{R}^{N}}|u(x)|^{2} d x\right)^{1 / 2} .
$$

And let

$$
E=\left\{u \in H^{s}\left(\mathbb{R}^{N}\right) \mid \int_{\mathbb{R}^{N}} A(x) u^{2} d x<+\infty\right\}
$$

be the Hilbert space equipped with norm

$$
\|u\|_{E}=\left(\|u\|_{H^{s}}^{2}+\int_{\mathbb{R}^{N}} A(x) u^{2} d x\right)^{1 / 2} .
$$

If $\lambda>0$, then it is equivalent to the norms

$$
\|u\|_{\lambda}=\left(\|u\|_{H^{s}}^{2}+\lambda \int_{\mathbb{R}^{N}} A(x) u^{2} d x\right)^{1 / 2}
$$

Thus $E$ is continuously embedded in $H^{s}\left(\mathbb{R}^{N}\right)$.

Remark 3. We know the embedding $H^{s}\left(\mathbb{R}^{N}\right) \hookrightarrow L^{\nu}\left(\mathbb{R}^{N}\right)$ is continuous; see [5] or [8]. So the embedding $E \hookrightarrow L^{\nu}\left(\mathbb{R}^{N}\right)$ is also continuous for any $v \in\left[2,2^{*}(s)\right]$.

Thanks to Remark 3, we can define the constant $S_{s}$ as in formula (14) and get that $S_{s}>0$.

Lemma 4. Let $u_{n} \in E$ be such that $\lambda_{n} \rightarrow \infty$ and $\left\|u_{n}\right\|_{\lambda_{n}}^{2}<C$. Then, there is a $u \in H_{0}^{s}(\Omega)$ such that, up to a subsequence, $u_{n} \rightarrow u$ in $L^{2}\left(\mathbb{R}^{N}\right)$.

Proof. If $u_{n} \rightarrow u$ strongly in $L^{2}\left(\mathbb{R}^{N}\right)$, we prove $u \in H_{0}^{s}(\Omega)$. Set $F_{m}=\{x:|x| \leq m, A(x) \geq 1 / m\}$, and $m \in \mathbb{N}$. For $n$ large enough that $\lambda_{n} \geq 1$, thanks to $\lambda_{n} \rightarrow \infty$. So $\left\|u_{n}\right\|_{E}^{2} \leq\left\|u_{n}\right\|_{\lambda_{n}}^{2}<$ $C$, we get

$$
\int_{F_{m}}\left|u_{n}\right|^{2} d x \leq m \int_{F_{m}} A(x)\left|u_{n}\right|^{2} d x \leq \frac{m C}{\lambda_{n}} \longrightarrow 0
$$


for every $m$. This implies that $u(x)=0$ for a.e. $x \in \mathbb{R}^{N} \backslash \Omega$. Hence, since $\partial \Omega$ is smooth, $u \in H_{0}^{s}(\Omega)$.

We will show that $u_{n} \rightarrow u$ strongly in $L^{2}\left(\mathbb{R}^{N}\right)$. Let $F=$ $\left\{x \in \mathbb{R}^{N}: A(x) \leq M_{0}\right\}$ with $M_{0}$ as in $(A 2)$, and let $F^{c}=$ $\mathbb{R}^{N} \backslash F$. Then

$$
\int_{F^{c}} u_{n}^{2} d x \leq \frac{1}{\lambda_{n} M_{0}} \int_{F^{c}} \lambda_{n} A(x) u_{n}^{2} d x \leq \frac{C}{\lambda_{n} M_{0}} \longrightarrow 0
$$

as $n \rightarrow \infty$. Setting $B_{R}^{c}=\mathbb{R}^{N} \backslash B_{R}$, where $B_{R}=\left\{x \in \mathbb{R}^{N}\right.$ : $|x| \leq R\}$, and choosing $r \in(1, N /(N-2 s))$, and $r^{\prime}=r /(r-1)$, we have

$$
\begin{gathered}
\int_{B_{R}^{c} \cap F}\left(u_{n}-u\right)^{2} d x \leq\left|u_{n}-u\right|_{2 r}^{2} L\left(B_{R}^{c} \cap F\right)^{1 / r^{\prime}} \\
\leq C_{1}\left\|u_{n}-u\right\|_{E}^{2} L\left(B_{R}^{c} \cap F\right)^{1 / r^{\prime}} \longrightarrow 0
\end{gathered}
$$

as $R \rightarrow \infty$, thanks to $(A 2)$. Since $u_{n} \rightarrow u$ in $L_{\text {loc }}^{2}\left(\mathbb{R}^{N}\right)$,

$$
\int_{B_{R}}\left(u_{n}-u\right)^{2} d x \longrightarrow 0
$$

as $n \rightarrow \infty$. By $u \in H_{0}^{s}(\Omega)$,

$$
\begin{aligned}
\int_{\mathbb{R}^{N}}\left(u_{n}-u\right)^{2} d x= & \int_{F^{c}} u_{n}^{2} d x+\int_{F}\left(u_{n}-u\right)^{2} d x \\
\leq & \int_{F^{c}} u_{n}^{2} d x+\int_{B_{R}^{c} \cap F}\left(u_{n}-u\right)^{2} d x \\
& +\int_{B_{R}}\left(u_{n}-u\right)^{2} d x \longrightarrow 0
\end{aligned}
$$

as $n \rightarrow \infty$. Thus $u_{n} \rightarrow u$ strongly in $L^{2}\left(\mathbb{R}^{N}\right)$.

We denote $A_{\lambda}:=(-\Delta)^{s}+\lambda A(x)$ and by $\langle\cdot, \cdot\rangle$ the $L^{2}$-inner product and write

$$
\begin{aligned}
\left\langle A_{\lambda} u, v\right\rangle= & \int_{\mathbb{R}^{2 N}} \frac{(u(x)-u(y))(v(x)-v(y))}{|x-y|^{N+2 s}} d x d y \\
& +\lambda \int_{\mathbb{R}^{N}} A(x) u v d x
\end{aligned}
$$

for $u, v \in E$. Set $a_{\lambda}:=\inf \sigma_{p}\left(A_{\lambda}\right)$, the infimum of the point spectrum of $A_{\lambda}$. Observe that

$$
0 \leq a_{\lambda}=\inf \left\{\left\langle A_{\lambda} u, u\right\rangle: u \in E,|u|_{2}=1\right\}
$$

and that $a_{\lambda}$ is nondecreasing in $\lambda$.

Lemma 5. For each $0<\mu<\mu_{1}(\Omega)$, there exists $\lambda(\mu)>0$ such that $a_{\lambda} \geq\left(\mu+\mu_{1}(\Omega)\right) / 2$ for $\lambda \geq \lambda(\mu)$. Consequently,

$$
c_{\mu}\|u\|_{\lambda}^{2} \leq\left\langle\left(A_{\lambda}-\mu\right) u, u\right\rangle
$$

for all $u \in E, \lambda \geq \lambda(\mu)$, where $c_{\mu}>0$ is a constant.
Proof. Assume, by contradiction, that there exists a sequence $\lambda_{n} \rightarrow \infty$ such that $a_{\lambda_{n}}<\left(\mu+\mu_{1}(\Omega)\right) / 2$ for all $n$ and $a_{\lambda_{n}} \rightarrow$ $c_{\lambda} \leq\left(\mu+\mu_{1}(\Omega)\right) / 2$. Let $u_{n} \in E$ be such that $\left|u_{n}\right|_{2}=1$ and $\left\langle\left(A_{\lambda_{n}}-a_{\lambda_{n}}\right) u_{n}, u_{n}\right\rangle \rightarrow 0$. Then

$$
\begin{aligned}
\left\|u_{n}\right\|_{\lambda_{n}}^{2}= & \int_{\mathbb{R}^{2 N}} \frac{\left|u_{n}(x)-u_{n}(y)\right|^{2}}{|x-y|^{N+2 s}} d x d y+\int_{\mathbb{R}^{N}}\left|u_{n}(x)\right|^{2} d x \\
& +\lambda_{n} \int_{\mathbb{R}^{N}} A(x)\left|u_{n}(x)\right|^{2} d x \\
= & \left\langle\left(A_{\lambda_{n}}-a_{\lambda_{n}}\right) u_{n}, u_{n}\right\rangle+\left(1+a_{\lambda_{n}}\right)\left|u_{n}\right|_{2}^{2} \\
\leq & 2\left(1+\mu_{1}(\Omega)\right)
\end{aligned}
$$

for all $n$ large. By Lemma 4 there is a $u \in H_{0}^{s}(\Omega)$ such that, up to a subsequence, $u_{n} \rightarrow u$ in $L^{2}\left(\mathbb{R}^{N}\right)$, and thus $|u|_{2}=1$. Using Fatou's theorem, we know

$$
\begin{gathered}
\int_{\mathbb{R}^{2 N}} \frac{|u(x)-u(y)|^{2}}{|x-y|^{N+2 s}} d x d y-c_{\lambda} \int_{\Omega}|u(x)|^{2} d x \\
\leq \lim _{n \rightarrow \infty} \inf \left(\int_{\mathbb{R}^{2 N}} \frac{\left|u_{n}(x)-u_{n}(y)\right|^{2}}{|x-y|^{N+2 s}} d x d y\right. \\
\left.\quad-a_{\lambda_{n}} \int_{\mathbb{R}^{N}}\left|u_{n}(x)\right|^{2} d x\right) \\
\leq \lim _{n \rightarrow \infty} \inf \left\langle\left(A_{\lambda_{n}}-a_{\lambda_{n}}\right) u_{n}, u_{n}\right\rangle=0 .
\end{gathered}
$$

Consequently,

$$
\int_{\mathbb{R}^{2 N}} \frac{|u(x)-u(y)|^{2}}{|x-y|^{N+2 s}} d x d y \leq c_{\lambda}<\frac{\left(\mu+\mu_{1}(\Omega)\right)}{2}<\mu_{1}(\Omega) .
$$

Since $\mu_{1}(\Omega)$ is the first eigenvalue of $(-\Delta)^{s}$ on $\Omega$ with boundary condition $u=0$, we have $\mu_{1}(\Omega) \leq$ $\int_{\mathbb{R}^{2 N}}\left(|u(x)-u(y)|^{2} /|x-y|^{N+2 s}\right) d x d y$. This is a contradic$\int_{\mathbb{R}^{2 N}}$ tion.

In the following, enlarging $\lambda(\mu)$ if necessary, we assume $\lambda(\mu) \geq \mu / M_{0}$; thus

$$
\lambda M_{0}-\mu \geq 0 \quad \forall \lambda \geq \lambda(\mu) .
$$

\section{The Proof of Theorem 1}

In this section we will finish the proof of Theorem 1 .

The critical points of $I_{\lambda}$ lie on the Nehari manifold

$$
M=\left\{u \in E \backslash\{0\}:\left\langle I_{\lambda}^{\prime}(u), u\right\rangle=0\right\} .
$$

Since $0<\mu<\mu_{1}(\Omega)$ and $2<2^{*}(s)$, the function $t \in \mathbb{R}_{+} \rightarrow$ $I_{\lambda}(t u)$ has a unique maximum point $t(u)>0$ and $t(u) u \in M$. Define

$$
c_{1}:=\inf _{M} I_{\lambda}
$$


and we observe that

$$
c_{1}=\inf _{u \in E, u \neq 0} \max _{t \geq 0} I_{\lambda}(t u) .
$$

From Lemma 5, the constant $c_{1}$ is positive. On the other hand, we define

$$
c=\inf _{\gamma \in \Gamma} \max _{t \in[0,1]} I_{\lambda}(\gamma(t))
$$

where

$$
\Gamma:=\left\{\gamma \in C([0,1], E): \gamma(0)=0, I_{\lambda}(\gamma(1))<0\right\} .
$$

Proposition 6. Consider $c=c_{1}$.

Proof. Proposition is proved, for instance, in [8, see Section 2].

$M$ is radially diffeomorphic $V=\left\{v \in E:|v|_{2^{*}(s)}=1\right\}$. For $u \in M$, the functional $I_{\lambda}$ is

$$
I_{\lambda}(u)=\frac{s}{N}\left\langle\left(A_{\lambda}-\mu\right) u, u\right\rangle=\frac{s}{N}|u|_{2^{*}(s)}^{2^{*}(s)} .
$$

So,

$$
c_{1}:=\inf _{u \in M} I_{\lambda}(u)=\inf _{v \in V} \frac{s}{N}\left\langle\left(A_{\lambda}-\mu\right) v, v\right\rangle^{N / 2 s} .
$$

We consider the functional

$$
\begin{aligned}
I_{\Omega}(u)= & \frac{1}{2} \int_{\mathbb{R}^{2 N}} \frac{|u(x)-u(y)|^{2}}{|x-y|^{N+2 s}} d x d y-\frac{1}{2} \mu \int_{\Omega}|u(x)|^{2} d x \\
& -\frac{1}{2^{*}(s)} \int_{\Omega}|u(x)|^{2^{*}(s)} d x \\
= & \frac{1}{2}\left\langle\left(A_{0}-\mu\right) u, u\right\rangle-\frac{1}{2^{*}(s)}|u|_{2^{*}(s)}^{2^{*}(s)}
\end{aligned}
$$

on $H_{0}^{s}(\Omega)$. Its Nehari manifold

$$
M_{\Omega}=\left\{u \in H_{0}^{s}(\Omega) \backslash\{0\}:\left\langle I_{\Omega}^{\prime}(u), u\right\rangle=0\right\}
$$

is radially diffeomorphic $V_{\Omega}=\left\{v \in H_{0}^{s}(\Omega):|v|_{2^{*}(s)}=1\right\}$. Set

$$
c(\Omega):=\inf _{u \in M_{\Omega}} I_{\Omega}(u)=\inf _{v \in V_{\Omega}} \frac{s}{N}\left\langle\left(A_{0}-\mu\right) v, v\right\rangle^{N / 2 s} .
$$

Proposition 7. If $0<\mu<\mu_{1}(\Omega)$ and $\lambda \geq \lambda(\mu)$, then

$$
\frac{s}{N}\left(c_{\mu} S_{s}\right)^{N / 2 s} \leq c<c(\Omega)<\frac{s}{N} S_{s}^{N / 2 s},
$$

where $S_{s}$ is defined in formula (14) and $c_{\mu}$ is given in Lemma 5.

Proof. By Lemma 5, $c_{\mu}\|v\|_{E}^{2} \leq c_{\mu}\|v\|_{\lambda}^{2} \leq\left\langle\left(A_{\lambda}-\mu\right) v, v\right\rangle$ for all $v \in E$. Taking infima over $v \in V$ gives the first inequality. Since $V_{\Omega} \subset V$ and $\left\langle A_{\lambda} v, v\right\rangle=\left\langle A_{0} v, v\right\rangle$ for $v \in V_{\Omega}$, it follows that $c \leq c(\Omega)$. By [6, see Section 7] and [10, see Section 8], we know $c(\Omega)<(s / N) S_{s}^{N / 2 s}$ and $c(\Omega)$ is achieved at some $u_{0}$. Thus $c<c(\Omega)$, because other $c$ would be also achieved at $u_{0}$ which vanishes outside $\Omega$, contradicting the maximum principle.

Hence, Proposition 7 is proved.
By definition of $c_{1}$ and Proposition 6, there exists a minimizing sequence for $I_{\lambda}$ on $M$, and we note $\left\{u_{j}\right\}$. By Ekeland's variational principle, we may assume that it is a Palais Smale sequence. So we have

$$
\begin{gathered}
I_{\lambda}\left(u_{j}\right) \longrightarrow c, \\
\sup \left\{\left|\left\langle I_{\lambda}^{\prime}\left(u_{j}\right), \varphi\right\rangle\right|: \varphi \in E,\|\varphi\|_{\lambda}=1\right\} \longrightarrow 0
\end{gathered}
$$

as $j \rightarrow+\infty$.

Proposition 8. $I_{\lambda}$ has at least one critical point with critical value $c$ for each $0<\mu<\mu_{1}(\Omega)$ and $\lambda \geq \lambda(\mu)$.

Proof. We proceed by steps.

Step 1. The sequence $\left\{u_{j}\right\}$ is bounded in $E$.

Proof. For any $j \in \mathbb{N}$ by (46) and (47) it easily follows that there exists $C_{1}>0$ such that

$$
\left|I_{\lambda}\left(u_{j}\right)\right| \leq C_{1}
$$

$$
\left|\left\langle I_{\lambda}^{\prime}\left(u_{j}\right), \frac{u_{j}}{\left\|u_{j}\right\|_{\lambda}}\right\rangle\right| \leq C_{1} \text {. }
$$

As a consequence of (48) we have

$$
\begin{aligned}
I_{\lambda}\left(u_{j}\right)-\frac{1}{2^{*}(s)}\left\langle I_{\lambda}^{\prime}\left(u_{j}\right), u_{j}\right\rangle & =\frac{s}{N}\left\langle\left(A_{\lambda}-\mu\right) u_{j}, u_{j}\right\rangle \\
& \leq C_{1}\left(1+\left\|u_{j}\right\|_{\lambda}\right) .
\end{aligned}
$$

By (49) and the definition of $I_{\lambda}$ we have

$$
\left\|u_{j}\right\|_{\lambda}^{2} \leq C_{2}\left(1+\left\|u_{j}\right\|_{\lambda}\right) .
$$

Thus $\left\{u_{j}\right\}$ is bounded in $E$.

Step 2. Problem (7) admits a solution $u_{\infty} \in E$.

Proof. By Step 1 and $E$ is a reflexive space, up to a subsequence, still denoted by $u_{j}$, there exists $u_{\infty} \in E$ such that $u_{j} \rightarrow u_{\infty}$ weakly in $E$; that is,

$$
\begin{aligned}
& \int_{\mathbb{R}^{2 N}} \frac{\left(u_{j}(x)-u_{j}(y)\right)(\varphi(x)-\varphi(y))}{|x-y|^{N+2 s}} d x d y \\
& \quad+\lambda \int_{\mathbb{R}^{N}} A(x) u_{j}(x) \varphi(x) d x \\
& \longrightarrow \int_{\mathbb{R}^{2 N}} \frac{\left(u_{\infty}(x)-u_{\infty}(y)\right)(\varphi(x)-\varphi(y))}{|x-y|^{N+2 s}} d x d y \\
& \quad+\lambda \int_{\mathbb{R}^{N}} A(x) u_{\infty}(x) \varphi(x) d x
\end{aligned}
$$

as $j \rightarrow+\infty$. Since Step 1 and Remark 3, we have that $u_{j}$ is bounded in $L^{2^{*}(s)}\left(\mathbb{R}^{N}\right)$. Since $L^{2^{*}(s)}\left(\mathbb{R}^{N}\right)$ is a reflexive space, up to a subsequence

$$
u_{j} \longrightarrow u_{\infty} \quad \text { weakly in } L^{2^{*}(s)}\left(\mathbb{R}^{N}\right)
$$


as $j \rightarrow+\infty$. While by Lemma 4 , up to a subsequence,

$$
\begin{gathered}
u_{j}(x) \longrightarrow u_{\infty}(x) \quad \text { in } L^{2}\left(\mathbb{R}^{N}\right), \\
u_{j} \longrightarrow u_{\infty} \quad \text { a.e. in } \mathbb{R}^{N}
\end{gathered}
$$

as $j \rightarrow+\infty$. By (52) and the fact that $\left|u_{j}\right|^{2^{*}(s)-2} u_{j}$ is bounded in $L^{2^{*}(s) /\left(2^{*}(s)-1\right)}\left(\mathbb{R}^{N}\right)$, we have

$$
\left|u_{j}\right|^{2^{*}(s)-2} u_{j} \longrightarrow\left|u_{\infty}\right|^{2^{*}(s)-2} u_{\infty}
$$$$
\text { weakly in } L^{2^{*}(s) /\left(2^{*}(s)-1\right)}\left(\mathbb{R}^{N}\right)
$$

as $j \rightarrow+\infty$.

Since (47) holds true, for any $\varphi \in E$

$$
\begin{aligned}
0 \longleftarrow & \left\langle I_{\lambda}^{\prime}\left(u_{j}\right), \varphi\right\rangle \\
= & \int_{\mathbb{R}^{2 N}} \frac{\left(u_{j}(x)-u_{j}(y)\right)(\varphi(x)-\varphi(y))}{|x-y|^{N+2 s}} d x d y \\
& +\lambda \int_{\mathbb{R}^{N}} A(x) u_{j}(x) \varphi(x) d x-\mu \int_{\mathbb{R}^{N}} u_{j}(x) \varphi(x) d x \\
& -\int_{\mathbb{R}^{N}}\left|u_{j}(x)\right|^{2^{*}(s)-2} u_{j}(x) \varphi(x) d x .
\end{aligned}
$$

Passing to the limit in this expression as $j \rightarrow+\infty$ and taking into account (51), (53), and (55), we get

$$
\begin{aligned}
\int_{\mathbb{R}^{2 N}} & \frac{\left(u_{\infty}(x)-u_{\infty}(y)\right)(\varphi(x)-\varphi(y))}{|x-y|^{N+2 s}} d x d y \\
& +\lambda \int_{\mathbb{R}^{N}} A(x) u_{\infty}(x) \varphi(x) d x-\mu \int_{\mathbb{R}^{N}} u_{\infty}(x) \varphi(x) d x \\
& -\int_{\mathbb{R}^{N}}\left|u_{\infty}(x)\right|^{2^{*}(s)-2} u_{\infty}(x) \varphi(x) d x=0
\end{aligned}
$$

for any $\varphi \in E$; that is, $u_{\infty}$ is a solution of problem (7).

Step 3. The following equality holds true:

$$
I_{\lambda}\left(u_{\infty}\right)=\frac{s}{N} \int_{\mathbb{R}^{N}}\left|u_{\infty}(x)\right|^{2^{*}(s)} d x \geq 0 .
$$

Proof. By Step 2, taking $\varphi=u_{\infty} \in E$ as a test function in (7), we have

$$
\begin{gathered}
\int_{\mathbb{R}^{2 N}} \frac{\left|u_{\infty}(x)-u_{\infty}(y)\right|^{2}}{|x-y|^{N+2 s}} d x d y+\lambda \int_{\mathbb{R}^{N}} A(x)\left|u_{\infty}(x)\right|^{2} d x \\
=\mu \int_{\mathbb{R}^{N}}\left|u_{\infty}(x)\right|^{2} d x+\int_{\mathbb{R}^{N}}\left|u_{\infty}(x)\right|^{2^{*}(s)} d x .
\end{gathered}
$$

So we get

$$
I_{\lambda}\left(u_{\infty}\right)=\frac{s}{N} \int_{\mathbb{R}^{N}}\left|u_{\infty}(x)\right|^{2^{*}(s)} d x \geq 0 .
$$

Hence, Step 3 is proved.

Now, we conclude the proof of Proposition 8.
We write $v_{j}:=u_{j}-u_{\infty}$, and then $v_{j} \rightarrow 0$ weakly in $E$. Moreover, since (54) holds true, by the Brézis-Lieb Lemma, we get

$$
\begin{aligned}
& \int_{\mathbb{R}^{2 N}} \frac{\left|u_{j}(x)-u_{j}(y)\right|^{2}}{|x-y|^{N+2 s}} d x d y+\lambda \int_{\mathbb{R}^{N}} A(x)\left|u_{j}(x)\right|^{2} d x \\
& =\int_{\mathbb{R}^{2 N}} \frac{\left|v_{j}(x)-v_{j}(y)\right|^{2}}{|x-y|^{N+2 s}} d x d y \\
& +\lambda \int_{\mathbb{R}^{N}} A(x)\left|v_{j}(x)\right|^{2} d x \\
& +\int_{\mathbb{R}^{2 N}} \frac{\left|u_{\infty}(x)-u_{\infty}(y)\right|^{2}}{|x-y|^{N+2 s}} d x d y \\
& +\lambda \int_{\mathbb{R}^{N}} A(x)\left|u_{\infty}(x)\right|^{2} d x+\circ(1), \\
& \int_{\mathbb{R}^{N}}\left|u_{j}(x)\right|^{2} d x=\int_{\mathbb{R}^{N}}\left|v_{j}(x)\right|^{2} d x \\
& +\int_{\mathbb{R}^{N}}\left|u_{\infty}(x)\right|^{2} d x+\circ(1), \\
& \int_{\mathbb{R}^{N}}\left|u_{j}(x)\right|^{2^{*}(s)} d x=\int_{\mathbb{R}^{N}}\left|v_{j}(x)\right|^{2^{*}(s)} d x \\
& +\left.u_{\infty}(x)\right|^{2^{*}(s)} d x+\circ(1), \\
& \int_{\mathbb{R}^{N}} \text { as } j \longrightarrow .+
\end{aligned}
$$

Then,

$$
\begin{aligned}
c \longleftarrow I_{\lambda}\left(u_{j}\right)= & \frac{1}{2} \int_{\mathbb{R}^{2 N}} \frac{\left|u_{j}(x)-u_{j}(y)\right|^{2}}{|x-y|^{N+2 s}} d x d y \\
& +\frac{1}{2} \lambda \int_{\mathbb{R}^{N}} A(x)\left|u_{j}(x)\right|^{2} d x \\
& -\frac{1}{2} \mu \int_{\mathbb{R}^{N}}\left|u_{j}(x)\right|^{2} d x \\
& -\frac{1}{2^{*}(s)} \int_{\mathbb{R}^{N}}\left|u_{j}(x)\right|^{2^{*}(s)} d x \\
= & \frac{1}{2} \int_{\mathbb{R}^{2 N}} \frac{\left|v_{j}(x)-v_{j}(y)\right|^{2}}{|x-y|^{N+2 s} d x d y} \\
& +\frac{1}{2} \lambda \int_{\mathbb{R}^{N}} A(x)\left|v_{j}(x)\right|^{2} d x \\
& +\frac{1}{2} \int_{\mathbb{R}^{2 N}} \frac{\left|u_{\infty}(x)-u_{\infty}(y)\right|^{2}}{|x-y|^{N+2 s}} d x d y \\
& +\frac{1}{2} \int_{\mathbb{R}^{N}} A(x)\left|u_{\infty}(x)\right|^{2} d x \\
& -\frac{1}{2} \mu \int_{\mathbb{R}^{N}}\left|u_{\infty}(x)\right|^{2} d x
\end{aligned}
$$




$$
\begin{aligned}
& \quad-\frac{1}{2} \mu \int_{\mathbb{R}^{N}}\left|v_{j}(x)\right|^{2} d x \\
& -\frac{1}{2^{*}(s)} \int_{\mathbb{R}^{N}}\left|v_{j}(x)\right|^{2^{*}(s)} d x \\
& \quad-\frac{1}{2^{*}(s)} \int_{\mathbb{R}^{N}}\left|u_{\infty}(x)\right|^{2^{*}(s)} d x+\circ(1) \\
& =I_{\lambda}\left(u_{\infty}\right)+\frac{1}{2} \int_{\mathbb{R}^{2 N}} \frac{\left|v_{j}(x)-v_{j}(y)\right|^{2}}{|x-y|^{N+2 s}} d x d y \\
& +\frac{1}{2} \int_{\mathbb{R}^{N}} A(x)\left|v_{j}(x)\right|^{2} d x \\
& \quad-\frac{1}{2} \mu \int_{\mathbb{R}^{N}}\left|v_{j}(x)\right|^{2} d x \\
& \quad-\frac{1}{2^{*}(s)} \int_{\mathbb{R}^{N}}\left|v_{j}(x)\right|^{2^{*}(s)} d x+\circ(1),
\end{aligned}
$$$$
\left\langle I_{\lambda}^{\prime}\left(u_{j}\right), u_{j}\right\rangle=\int_{\mathbb{R}^{2 N}} \frac{\left|u_{j}(x)-u_{j}(y)\right|^{2}}{|x-y|^{N+2 s}} d x d y
$$$$
+\lambda \int_{\mathbb{R}^{N}} A(x)\left|u_{j}(x)\right|^{2} d x
$$$$
-\mu \int_{\mathbb{R}^{N}}\left|u_{j}(x)\right|^{2} d x-\int_{\mathbb{R}^{N}}\left|u_{j}(x)\right|^{2^{*}(s)} d x
$$$$
=\int_{\mathbb{R}^{2 N}} \frac{\left|v_{j}(x)-v_{j}(y)\right|^{2}}{|x-y|^{N+2 s}} d x d y
$$$$
+\lambda \int_{\mathbb{R}^{N}} A(x)\left|v_{j}(x)\right|^{2} d x
$$$$
+\int_{\mathbb{R}^{2 N}} \frac{\left|u_{\infty}(x)-u_{\infty}(y)\right|^{2}}{|x-y|^{N+2 s}} d x d y
$$$$
+\lambda \int_{\mathbb{R}^{N}} A(x)\left|u_{\infty}(x)\right|^{2} d x
$$$$
-\mu \int_{\mathbb{R}^{N}}\left|u_{\infty}(x)\right|^{2} d x-\mu \int_{\mathbb{R}^{N}}\left|v_{j}(x)\right|^{2} d x
$$$$
-\int_{\mathbb{R}^{N}}\left|v_{j}(x)\right|^{2^{*}(s)} d x
$$$$
-\int_{\mathbb{R}^{N}}\left|u_{\infty}(x)\right|^{2^{*}(s)} d x+\circ(1)
$$$$
=\left\langle I_{\lambda}^{\prime}\left(u_{\infty}\right), u_{\infty}\right\rangle
$$$$
+\int_{\mathbb{R}^{2 N}} \frac{\left|v_{j}(x)-v_{j}(y)\right|^{2}}{|x-y|^{N+2 s}} d x d y
$$$$
+\lambda \int_{\mathbb{R}^{N}} A(x)\left|v_{j}(x)\right|^{2} d x
$$

$$
\begin{aligned}
& -\mu \int_{\mathbb{R}^{N}}\left|v_{j}(x)\right|^{2} d x \\
& -\int_{\mathbb{R}^{N}}\left|v_{j}(x)\right|^{2^{*}(s)} d x+\circ(1) .
\end{aligned}
$$

$\operatorname{By}\left\langle I_{\lambda}^{\prime}\left(u_{\infty}\right), u_{\infty}\right\rangle=0$ and $\left\langle I_{\lambda}^{\prime}\left(v_{j}\right), v_{j}\right\rangle \rightarrow 0$, we get

$$
\begin{gathered}
\int_{\mathbb{R}^{2 N}} \frac{\left|v_{j}(x)-v_{j}(y)\right|^{2}}{|x-y|^{N+2 s}} d x d y \\
+\lambda \int_{\mathbb{R}^{N}} A(x)\left|v_{j}(x)\right|^{2} d x-\mu \int_{\mathbb{R}^{N}}\left|v_{j}(x)\right|^{2} d x \longrightarrow b, \\
\int_{\mathbb{R}^{N}}\left|v_{j}(x)\right|^{2^{*}(s)} d x \longrightarrow b .
\end{gathered}
$$

As in the proof of Lemma 4 one shows that

$$
\int_{F}\left|v_{j}(x)\right|^{2} d x \longrightarrow 0
$$

as $j \rightarrow \infty$, where $F=\left\{x \in \mathbb{R}^{N}: A(x) \leq M_{0}\right\}$. Let $F^{c}=$ $\mathbb{R}^{N} \backslash F$. Then, by (34),

$$
\begin{aligned}
S_{s}\left\|v_{j}\right\|_{L^{2^{*}(s)}\left(\mathbb{R}^{N}\right)}^{2} \leq & \int_{\mathbb{R}^{2 N}} \frac{\left|v_{j}(x)-v_{j}(y)\right|^{2}}{|x-y|^{N+2 s}} d x d y \\
\leq & \int_{\mathbb{R}^{2 N}} \frac{\left|v_{j}(x)-v_{j}(y)\right|^{2}}{|x-y|^{N+2 s}} d x d y \\
& +\int_{F^{c}}(\lambda A(x)-\mu)\left|v_{j}(x)\right|^{2} d x \\
\leq & \left\langle\left(A_{\lambda}-\mu\right) v_{j}, v_{j}\right\rangle+\mu \int_{F}\left|v_{j}(x)\right|^{2} d x \\
= & \left\langle\left(A_{\lambda}-\mu\right) v_{j}, v_{j}\right\rangle+\circ(1) .
\end{aligned}
$$

Passing to the limit yields $b \geq S_{s} b^{2 / 2^{*}(s)}$. Either $b=0$ or $b \geq$ $S_{s}^{N / 2 s}$. If $b=0$, the proof is complete. Assuming $b \geq S_{s}^{N / 2 s}$, we obtain from Step 3, (45), and (62) that

$$
\frac{s}{N} S_{s}^{N / 2 s} \leq\left(\frac{1}{2}-\frac{1}{2^{*}(s)}\right) b=c<\frac{s}{N} S_{s}^{N / 2 s}
$$

which is a contradiction. Thus $b=0$, and

$$
\left\|u_{j}-u_{\infty}\right\|_{\lambda} \longrightarrow 0
$$

as $j \rightarrow+\infty$. This ends the proof of Proposition 8 .

We have finished the proof of Theorem 1 by Proposition 8 . 


\section{The proof of Theorem 2}

Proof of Theorem 2. Let $\left(u_{n}\right)$ be a sequence of solutions of (4) such that $0<\mu<\mu_{1}(\Omega), \lambda_{n} \rightarrow \infty$, and $N I_{\lambda_{n}}\left(u_{n}\right)=\left\langle\left(A_{\lambda_{n}}-\right.\right.$ $\left.\mu) u_{n}, u_{n}\right\rangle \rightarrow N c<s S_{s}^{N / 2 s}$. Then, by Lemma 4 , there is a $u \in$ $H_{0}^{s}(\Omega)$ such that, up to a subsequence, $u_{n} \rightarrow u$ in $E$. By $u_{n}$ that is a solution of (4), we have

$$
\begin{aligned}
& \int_{\mathbb{R}^{2 N}} \frac{\left(u_{n}(x)-u_{n}(y)\right)(\varphi(x)-\varphi(y))}{|x-y|^{N+2 s}} d x d y \\
& \quad+\lambda_{n} \int_{\mathbb{R}^{N}} A(x) u_{n}(x) \varphi(x) d x-\mu \int_{\mathbb{R}^{N}} u_{n}(x) \varphi(x) d x \\
& =\int_{\mathbb{R}^{N}}\left|u_{n}(x)\right|^{2^{*}(s)-2} u_{n}(x) \varphi(x) d x
\end{aligned}
$$

for any $\varphi \in E$. If $\varphi \in H_{0}^{s}(\Omega)$, then $\lambda_{n} \int_{\mathbb{R}^{N}} A(x) u_{n}(x) \varphi(x) d x=$ 0 for all $n$, so letting $n \rightarrow \infty$ we obtain

$$
\begin{aligned}
& \int_{\mathbb{R}^{2 N}} \frac{(u(x)-u(y))(\varphi(x)-\varphi(y))}{|x-y|^{N+2 s}} d x d y \\
& -\mu \int_{\mathbb{R}^{N}} u(x) \varphi(x) d x=\int_{\mathbb{R}^{N}}|u(x)|^{2^{*}(s)-2} u(x) \varphi(x) d x
\end{aligned}
$$

for any $\varphi \in H_{0}^{s}(\Omega)$. So, $u$ is a solution of (13). We write $v_{n}:=$ $u_{n}-u$. Then, $v_{n} \rightarrow 0$ in $L^{2}\left(\mathbb{R}^{N}\right)$.

Since $A(x)=0$ for $x \in \Omega$, we get

$$
\left\langle\left(A_{\lambda_{n}}-\mu\right) u_{n}, u_{n}\right\rangle=\left\langle\left(A_{0}-\mu\right) u, u\right\rangle+\left\langle\left(A_{\lambda_{n}}-\mu\right) v_{n}, v_{n}\right\rangle .
$$

By $v_{n} \rightarrow 0$ in $E$ and the Brézis-Lieb Lemma, we have

$$
\begin{aligned}
\int_{\mathbb{R}^{N}}\left|u_{n}(x)\right|^{2^{*}(s)} d x \\
\quad=\int_{\mathbb{R}^{N}}|u(x)|^{2^{*}(s)} d x+\int_{\mathbb{R}^{N}}\left|v_{n}(x)\right|^{2^{*}(s)} d x+\circ(1) .
\end{aligned}
$$

So, we can get

$$
\left\langle\left(A_{\lambda_{n}}-\mu\right) v_{n}, v_{n}\right\rangle-\int_{\mathbb{R}^{N}}\left|v_{n}(x)\right|^{2^{*}(s)} d x=\circ(1) .
$$

We claim that $\int_{\mathbb{R}^{N}}\left|v_{n}(x)\right|^{2^{*}(s)} d x \quad \rightarrow \quad 0$. Assume $\int_{\mathbb{R}^{N}}\left|v_{n}(x)\right|^{2^{*}(s)} d x \rightarrow b>0$. Then,

$$
\begin{aligned}
S_{s}\left(\int_{\mathbb{R}^{N}}\left|v_{n}(x)\right|^{2^{*}(s)} d x\right)^{2 / 2^{*}(s)} & \leq \int_{\mathbb{R}^{2 N}} \frac{\left|v_{n}(x)-v_{n}(y)\right|^{2}}{|x-y|^{N+2 s}} d x d y \\
& \leq\left\langle\left(A_{\lambda_{n}}-\mu\right) v_{n}, v_{n}\right\rangle \\
& =\int_{\mathbb{R}^{N}}\left|v_{n}(x)\right|^{2^{*}(s)} d x+\circ(1),
\end{aligned}
$$

thanks to (73). It follows that

$$
\begin{aligned}
S_{s} & \leq\left(\int_{\mathbb{R}^{N}}\left|v_{n}(x)\right|^{2^{*}(s)} d x\right)^{\left(2^{*}(s)-2\right) / 2^{*}(s)}+\circ(1) \\
& \leq\left(\int_{\mathbb{R}^{N}}\left|u_{n}(x)\right|^{2^{*}(s)} d x\right)^{\left(2^{*}(s)-2\right) / 2^{*}(s)}+\circ(1), \\
S_{s}^{N / 2 s} & \leq \lim _{n \rightarrow \infty} \int_{\mathbb{R}^{N}}\left|u_{n}(x)\right|^{2^{*}(s)} d x \\
& =\lim _{n \rightarrow \infty}\left\langle\left(A_{\lambda_{n}}-\mu\right) u_{n}, u_{n}\right\rangle=N c<s S_{s}^{N / 2 s} .
\end{aligned}
$$

This is a contradiction. Thus $\int_{\mathbb{R}^{N}}\left|v_{n}(x)\right|^{2^{*}(s)} d x \rightarrow 0$ and $\left\langle\left(A_{\lambda_{n}}-\mu\right) v_{n}, v_{n}\right\rangle \rightarrow 0$, by (73). Hence, by (71)

$$
\lim _{n \rightarrow \infty}\left\langle\left(A_{\lambda_{n}}-\mu\right) u_{n}, u_{n}\right\rangle=\left\langle\left(A_{0}-\mu\right) u, u\right\rangle \text {. }
$$

Since $u_{n}=v_{n}$ in $\mathbb{R}^{N} \backslash \Omega$ and $A(x)=0$ for $x \in \Omega$,

$$
\begin{aligned}
\int_{\mathbb{R}^{N}} A(x)\left|u_{n}(x)\right|^{2} d x & \leq \int_{\mathbb{R}^{N}} \lambda_{n} A(x)\left|u_{n}(x)\right|^{2} d x \\
& =\int_{\mathbb{R}^{N}} \lambda_{n} A(x)\left|v_{n}(x)\right|^{2} d x \\
& \leq\left\langle\left(A_{\lambda_{n}}-\mu\right) v_{n}, v_{n}\right\rangle .
\end{aligned}
$$

Therefore, $\int_{\mathbb{R}^{N}} A(x)\left|u_{n}(x)\right|^{2} d x \rightarrow 0$ and (76) implies that $u_{n} \rightarrow u$ in $E$.

\section{Acknowledgment}

This paper is supported by the National Nature Science Foundation of China (11271331).

\section{References}

[1] M. Clapp and Y. Ding, "Positive solutions of a Schrödinger equation with critical nonlinearity," Zeitschrift für Angewandte Mathematik und Physik, vol. 55, no. 4, pp. 592-605, 2004.

[2] M. Clapp and Y. Ding, "Minimal nodal solutions of a Schrödinger equation with critical nonlinearity and symmetric potential," Differential and Integral Equations, vol. 16, no. 8, pp. 981-992, 2003.

[3] A. Floer and A. Weinstein, "Nonspreading wave packets for the cubic Schrödinger equation with a bounded potential," Journal of Functional Analysis, vol. 69, no. 3, pp. 397-408, 1986.

[4] R. Servadei and E. Valdinoci, “The Brézis-Nirenberg result for the fractional Laplacian," to appear in Transactions of the American Mathematical Society.

[5] E. Di Nezza, G. Palatucci, and E. Valdinoci, "Hitchhiker's guide to the fractional Sobolev spaces," Bulletin des Sciences Mathématiques, vol. 136, no. 5, pp. 521-573, 2012.

[6] R. Servadei, "The Yamabe equation in a non-local setting," Advances in Nonlinear Analysis, vol. 2, no. 3, pp. 235-270, 2013.

[7] R. Servadei and E. Valdinoci, "Fractional Laplacian equations with critical sobolev exponent," submitted.

[8] P. Felmer, A. Quaas, and J. G. Tan, "Positive solutions of nonlinear Schrödinger equation with fractional Laplacian," Proceedings of the Royal Society of Edinburgh, vol. 142, no. 6, pp. 12371262, 2012. 
[9] R. Servadei and E. Valdinoci, "Variational methods for nonlocal operators of elliptic type," Discrete and Continuous Dynamical Systems, vol. 33, no. 5, pp. 2105-2137, 2013.

[10] R. Servadei and A. Brézis-Nirenberg, "A Brezis-Nirenberg result for non-local critical equations in low dimension," Communications on Pure and Applied Analysis, vol. 12, no. 6, pp. 2445-2464, 2013. 


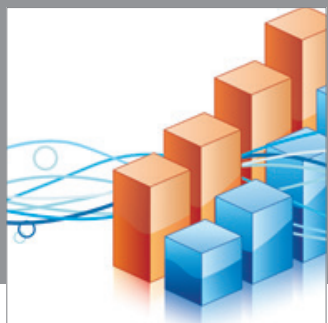

Advances in

Operations Research

mansans

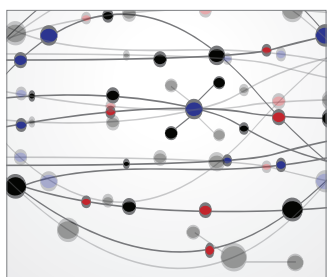

The Scientific World Journal
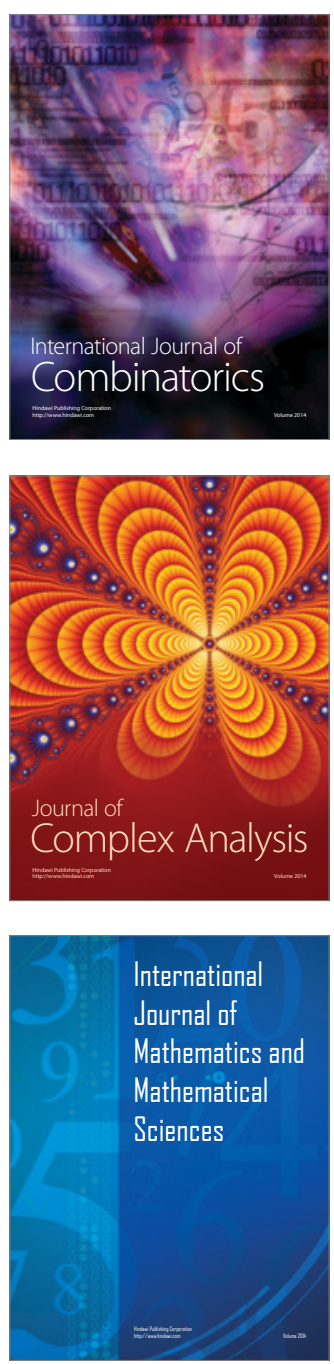
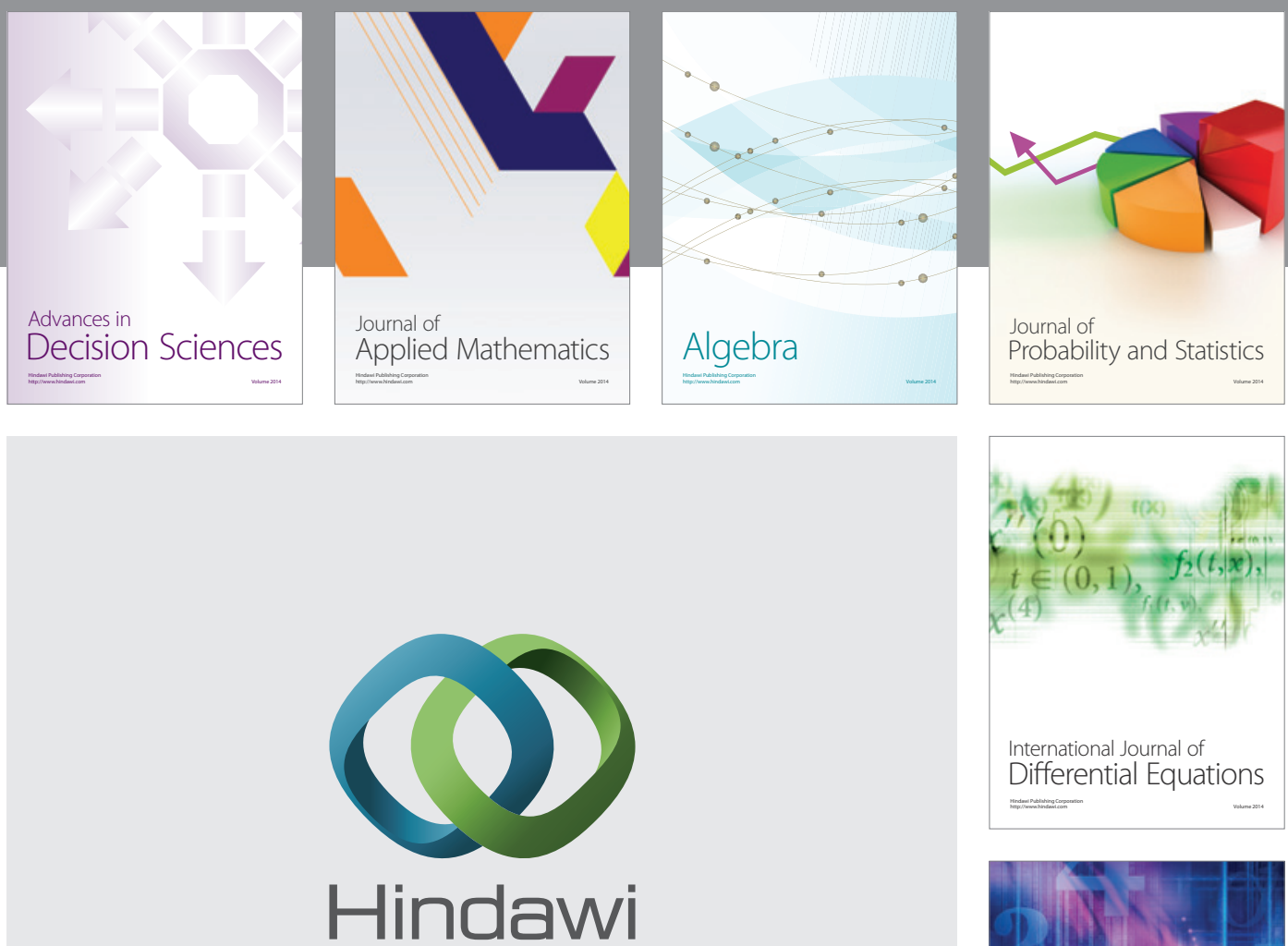

Submit your manuscripts at http://www.hindawi.com
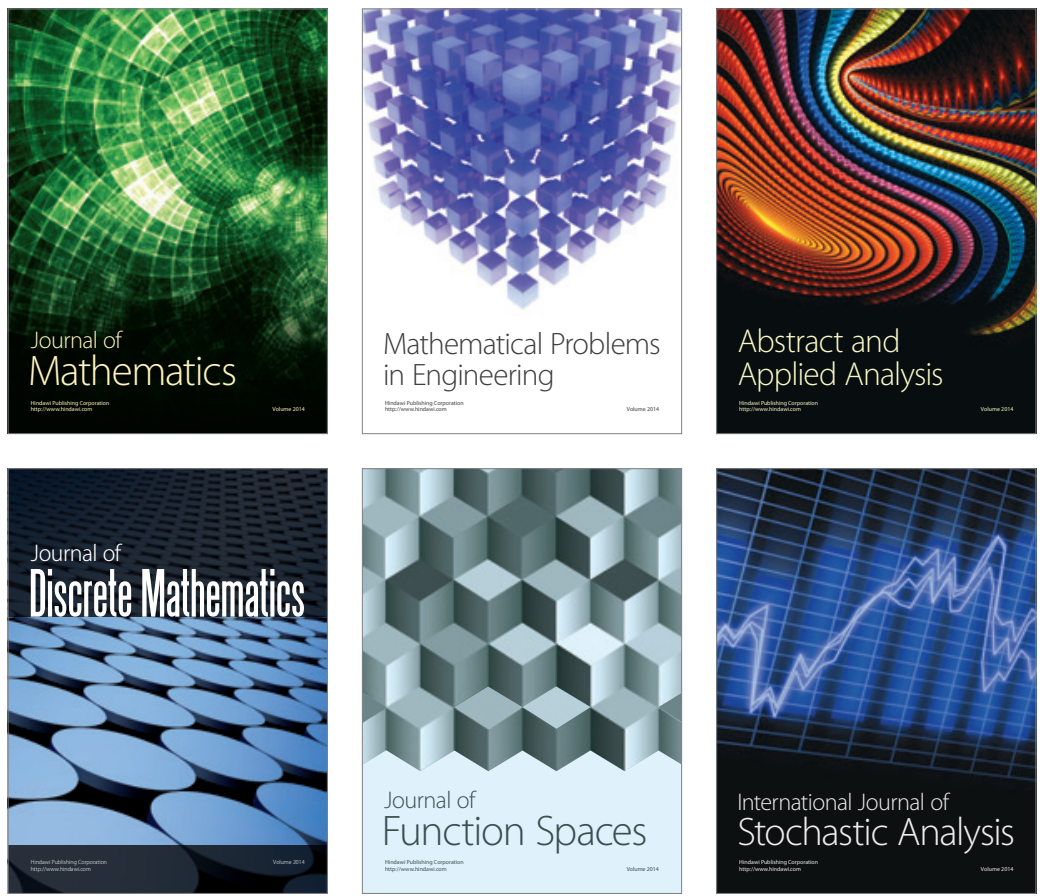

Journal of

Function Spaces

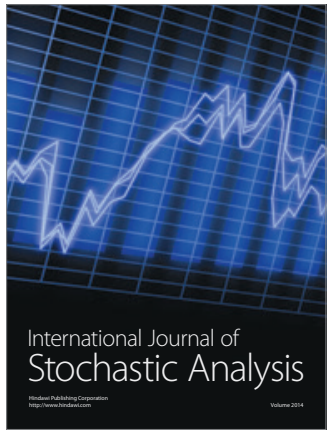

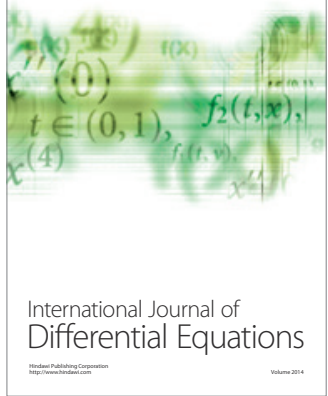
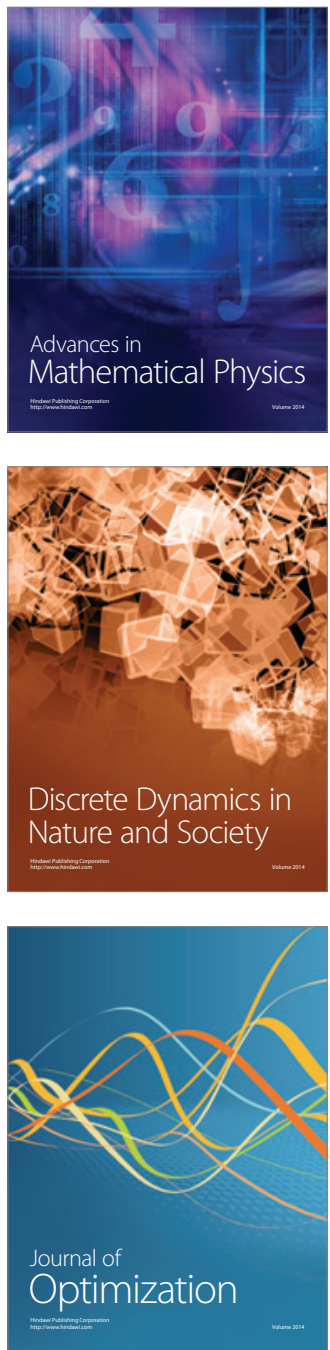